\title{
Prevalência da administração de medicamentos sem a orientação do Médico Veterinário em animais de companhia na Cidade de Areia - PB
}

\author{
Prevalence of drug administration without the guidance of the Veterinarian in companion animals \\ in the City of Areia - PB \\ Prevalencia de la administración de medicamentos sin la orientación del Veterinario em animales \\ de compañía em la Ciudad de Areia - PB
}

Recebido: 28/06/2021 | Revisado: 04/07/2021 | Aceito: 05/07/2021 | Publicado: 17/07/2021

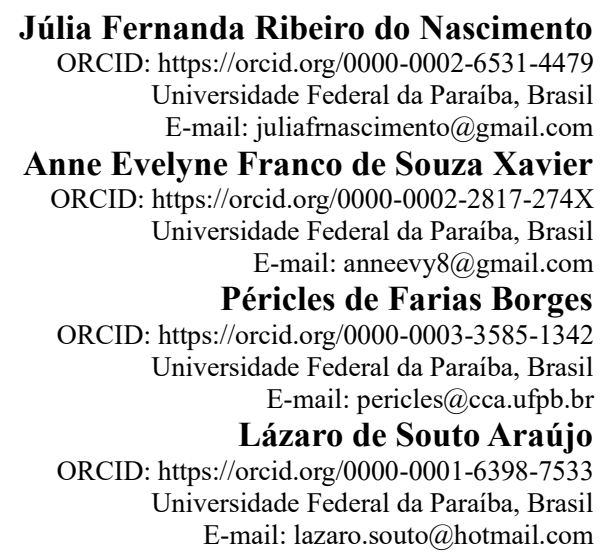

\begin{abstract}
Resumo
Os animais de companhia têm sido tratados como membros da família, estão habituados ao ambiente domiciliar, sendo criados como seres humanos e por isso foi criado um vínculo afetivo entre tutor e animal. Por esse motivo estão sujeitos a medicação sem prescrição de um profissional habilitado, visto que essa é uma prática comum dos humanos. Muitos tutores desconhecem o potencial tóxico que alguns fármacos apresentam se administrados em doses erradas. A dosagem incorreta de um medicamento pode levar ao diagnóstico inexato de doenças graves, problemas crônicos como insuficiência renal e hepática e, principalmente a intoxicações. Portanto, objetivou-se nessa pesquisa avaliar a prevalência dos casos de administração de medicamentos sem a orientação do médico veterinário, em animais de companhia, na cidade de Areia - PB, bem como identificar quais são os fármacos mais utilizados e o conhecimento da população a respeito dos riscos da automedicação e do potencial tóxico que alguns fármacos apresentam. Foram aplicados 46 questionários online, através do software Google Forms ${ }^{\circledR}$ e 8 questionários presenciais com tutores de animais, residentes na cidade de Areia - PB, no período de agosto a setembro de 2019, totalizando 54 questionários. A análise foi realizada com base nas respostas dos participantes que atenderam aos critérios de inclusão. Apesar de grande parte dos entrevistados conhecerem os riscos da automedicação, uma alta parcela dessas pessoas ainda realiza essa prática. Os resultados confirmam a necessidade de mais esclarecimento à população e leis em defesa da saúde animal. Palavras-chave: Animais; Automedicação; Intoxicação; Veterinário.
\end{abstract}

\footnotetext{
Abstract

Companion animals have been treated as family members, they are used to the home environment, being raised as human beings, and therefore an affective bond was created between guardian and animal. For this reason, they are subject to medication without a prescription from a qualified professional, as this is a common practice of humans. Many tutors are unaware of the toxic potential that some drugs have if administered in wrong doses. Incorrect dosage of a drug can lead to an inaccurate diagnosis of serious diseases, chronic problems such as kidney and liver failure, and especially poisoning. Therefore, the objective of this research was to evaluate the prevalence of medication administration cases without the guindace of the veterinarian, in pets, in the city of Areia - PB, Brazil, as well as identify which are the most used drugs and the knowledge of the population about the risks of selfmedication and the toxic potential that some drugs present. 46 online questionnaires were applied through Google Forms® software and 8 faceto-face questionnaires with animal tutors, living in the city of Areia - PB, Brazil, from August to September 2019, totaling 54 questionnaires. The analysis was performed based on the responses of participants who met the inclusion criteria. Although a large part of the interviewees are aware of the risks of self-medication, a high proportion of these
} 
people still carry out this practice. The results confirm the need for more clarification to the population and laws in defense of animal health.

Keywords: Animals; Intoxication; Self-medication; Veterinary.

\begin{abstract}
Resumen
Los animales de compañía han sido tratados como miembros de la familia, están acostumbrados al ambiente del hogar, siendo criados como seres humanos, por lo que se creó un vínculo afectivo entre el guardián y el animal. Por esta razón, están sujetos a medicación sin receta de un profesional calificado, ya que esta es una práctica común de los humanos. Muchos tutores desconecen el potencial tóxico que tienen algunos medicamentos si se administran en dosis incorrectas. La dosificación incorrecta de un medicamento puede conducir a un diagnóstico inexacto de enfermedades graves, problemas crónicos como insuficiencia renal y hepática, y especialmente intoxicación. Por lo tanto, el objetivo de esta investigación fue evaluar la prevalencia de casos de administración de medicamentos sin orientación del veterinario, en animales de compañía, en la ciudad de Areia - PB, así como identificar cuáles son los medicamentos más utilizados y el conocimiento de la población sobre los riesgos de la automedicación y el potencial tóxico que presentam algunos fármacos. Se aplicaron 46 cuestionarios en línea, a través del software Google Forms ${ }^{\circledR}$ y 8 cuestionarios presenciales com tutores de animales, residentes en la ciudad de Areia - PB, en el período de agosto a septiembre de 2019, totalizando 54 cuestionarios. El análisis se realizó en base a las respuestas de los participantes que cumplieron com los criterios de inclusión. Aunque la mayoría de los encuestados conocen los riesgos de la automedicación, una alta proporción de estas personas aún realiza esta práctica. Los resultados confirman la necesidad de mayor aclaración a la población y leyes en defensa de la salud animal.
\end{abstract}

Palabras clave: Animales; Automedicación; Intoxicación; Veterinario.

\title{
1. Introdução
}

A ação que um doente ou seu responsável tem de usar um produto no tratamento de uma doença ou aliviar seus sintomas, sem a orientação de um especialista de saúde habilitado, é definida pela Agência Nacional de Vigilância Sanitária (ANVISA) como automedicação, Agência Nacional de Vigilância Sanitária [ANVISA] (2007).

Frequentemente tutores têm medicado seus animais sem a orientação ou prescrição de um médico veterinário. Muitos desconhecem o potencial tóxico que alguns fármacos apresentam se administrados em doses erradas. Alguns fármacos, que podem intoxicar cães em determinadas doses, tem a capacidade de intoxicar gatos em doses menores (Anjos \& Brito, 2009). Outros fatores que influenciam na intoxicação medicamentosa, além da espécie, são a raça, idade, sexo, condição corporal, histórico médico pregresso e status vacinal (Riboldi, 2010).

Dentre os medicamentos que são comumente administrados erroneamente, sendo as principais causas de intoxicação em animais domésticos, estão os anti-inflamatórios não-esteroidais (AINES), principalmente o paracetamol, e produtos de uso tópico (Xavier, Maruo, \& Spinosa, 2008). Por exemplo, o paracetamol tem ação analgésica, antipirética e possui baixo potencial anti-inflamatório, o mesmo não é indicado para a administração em gatos, pois nesses animais não ocorre corretamente a conjugação com a enzima hepática glicuronil-transferase, essencial na eliminação deste AINE (Tasaka, 2011).

Segundo o Instituto Brasileiro de Geografia e Estatística (IBGE), através da Pesquisa Nacional de Saúde realizada no ano de 2019, o Brasil dispõe de mais de 46\% de cães e mais de 19\% de gatos em domicílios, Instituto Brasileiro de Geografia e Estatística [IBGE] (2020). Esses dados indicam quanto o ser humano tem se aproximado dos animais de companhia e atribuído a eles o papel de membro da família, por esse motivo o pet está sujeito a ser tratado como um humano e ao apresentar alguma doença é submetido à automedicação o que consequentemente, leva o mesmo a uma intoxicação medicamentosa, Zielke et al. (2018).

Com base nessas informações, surgiram hipóteses de que a prática da automedicação é maior quando a pessoa desconhece seus riscos e o potencial tóxico que alguns fármacos apresentam. Além disso, a falta de conhecimento da população sobre os riscos da automedicação pode contribuir para o aumento do número de casos dessa prática e possíveis intoxicações medicamentosas (Santos, 2014). Por isso se torna necessário a obtenção de dados a respeito da prevalência de casos de automedicação em animais de companhia e sobre o conhecimento da população sobre a prática, sendo esse um importante parâmetro para traçar estratégias de educação em saúde animal e campanhas de conscientização a respeito dos riscos da 
automedicação, que são formas efetivas de se diminuir a proporção dessa prática (Benedito, Albuquerque, Taffarel \& BastosPereira, 2017a). A partir disso, o presente trabalho tem o objetivo de avaliar a prevalência dos casos de administração de medicamentos sem a orientação do médico veterinário em animais de companhia na cidade de Areia - PB.

\section{Metodologia}

O estudo em pauta configura-se como uma pesquisa descritiva, transversal, exploratória, observacional, de campo e quantitativa. A pesquisa foi realizada através da aplicação de questionários submetidos na plataforma online Google Forms ${ }^{\circledR}$, um aplicativo de pesquisas pertencente a empresa Google que permite a elaboração de questionários para serem computados de forma colaborativa (Mota, 2019). Os questionários foram submetidos à usuários de internet residentes na cidade de Areia, estado da Paraíba. Juntamente com os questionários online, foram aplicados questionários presenciais com tutores de animais, também residentes na cidade de Areia - PB. Anteriormente a aplicação dos questionários, houve a submissão através da Plataforma Brasil ao Comitê de Ética e Pesquisa com Seres Humanos do Centro de Ciências da Saúde (CEP/CCS) da Universidade Federal da Paraíba (UFPB), sendo iniciada a coleta de dados após aprovação

O questionário constituía-se por questões de múltipla escolha e discursivas, contendo perguntas relacionadas ao uso de medicamentos sem a indicação de um médico veterinário, os fármacos mais comumente utilizados e se os participantes possuíam algum conhecimento sobre os riscos que a prática da automedicação pode causar, e relacionado ao potencial tóxico que alguns fármacos podem apresentar. Foram aplicados 46 questionários online, através do software Google Forms $®$ e 8 questionários presenciais, no período de agosto a setembro de 2019, totalizando 54 questionários. Destes, 2 foram excluídos por não cumprirem as medidas de inclusão da pesquisa, sendo assim a análise foi realizada com base nas respostas dos 52 participantes que atenderam aos critérios de inclusão.

Foram incluídos na pesquisa os cidadãos brasileiros maiores de 18 anos, residentes no município de Areia no estado da Paraíba e que possuíam algum animal de companhia. Pessoas que não eram residentes no município de Areia - PB, as menores de 18 anos, as que não possuíam nenhum animal de companhia, bem como as que não concluíram o processo de preenchimento do protocolo da pesquisa, foram excluídas da pesquisa.

De início, o objetivo seria atingir o maior número de pessoas, com a finalidade de conseguir uma amostra estatisticamente representativa. Porém, o objetivo foi limitado com o surgimento de dois problemas práticos: a falta de registros relacionados a quantidade de pessoas tutoras de animais de companhia para a região avaliada, o que dificulta a mensuração do tamanho efetivo da população; e a dificuldade de abranger toda a população que compõe a cidade avaliada, acima de tudo presencialmente. Neste caso, foi empregado o sistema de amostragem não probabilística por acessibilidade, que se justifica quando o pesquisador não possui dados estatísticos consideráveis sobre a população estudada e é necessário para auxiliar a execução da pesquisa (Aaker, Kumar \& Day, 1995).

A coleta de dados teve início no mês de agosto de 2019 até o mês de setembro do mesmo ano. Seguida das etapas de análise e discussão de resultados, que ocorreram no mês de outubro de 2019. A análise dos dados realizou-se de acordo com as informações obtidas nos questionários. Os dados foram analisados e processados no software Microsoft Excel 2010 para disposição das variáveis. E a análise estatística foi realizada através de distribuição de frequência.

\section{Resultados e Discussão}

Primeiramente foi analisado o perfil socioeconômico dos participantes (Tabela 1). Dentre os entrevistados 34 (65,4\%) eram do sexo feminino. Relacionado à faixa etária, 28 (53,8\%) possuíam entre 18 e 25 anos, 16 (30,8\%) entre 26 e 35 anos, 7 $(13,5 \%)$ de 36 a 50 anos e $1(1,9 \%)$ acima de 60 anos. A respeito do local de residência, 48 (92,3\%) residiam em zona urbana e 
4 (7,7\%) em zona rural. Em relação ao nível de escolaridade, 4 (7,7\%) não tinham completado o ensino médio, 9 (17,3\%) haviam completado o ensino médio e a maioria estavam cursando ou haviam concluído o ensino superior e pós-graduação, correspondendo a 39 (75\%) dos participantes. Em relação à renda familiar, 1 (1,9\%) não possuía nenhuma renda, a maioria, 42 (80,8\%) recebia até 3 salários mínimos e 9 (17,3\%) recebiam até 6 salários mínimos. Uma pesquisa conduzida pelo Serviço de Proteção ao Crédito Brasil e pela Confederação Nacional de Dirigentes Lojistas aponta que fatores como gênero ou classe social não influenciam na composição do perfil das pessoas que possuem pets, 50,2\% são homens, enquanto $54,4 \%$ pertencem à classe C/D/E, essa classe corresponde as pessoas que possuem renda familiar até 3 salários mínimos, e os outros 45,6\% à classe A/B, correspondente a classe de pessoas que possuem renda familiar de mais de 6 salários mínimos. Foi também observado que a maior parte (57,7\%) corresponde às faixas etárias de 25 a 44 anos, Serviço de Proteção ao Crédito Brasil [SPC Brasil] (2017); Fundação Getúlio Vargas Social [FGV Social] (2014).

Tabela 1. Perfil socioeconômico dos participantes.

\begin{tabular}{|c|c|c|c|}
\hline Variável & Categoria & $\mathbf{n}$ & $\%$ \\
\hline \multirow[t]{2}{*}{ Sexo } & Feminino & 34 & 65,4 \\
\hline & Masculino & 18 & 34,6 \\
\hline \multirow[t]{4}{*}{ Faixa etária } & 18 a 25 anos & 28 & 53,8 \\
\hline & 26 a 35 anos & 16 & 30,8 \\
\hline & 36 a 50 anos & 7 & 13,5 \\
\hline & Acima de 60 anos & 1 & 1,9 \\
\hline \multirow[t]{2}{*}{ Local de residência } & Zona urbana & 48 & 92,3 \\
\hline & Zona rural & 4 & 7,7 \\
\hline \multirow[t]{6}{*}{ Nível de escolaridade } & Fundamental incompleto & 3 & 5,8 \\
\hline & Médio incompleto & 1 & 1,9 \\
\hline & Médio completo & 9 & 17,3 \\
\hline & Superior incompleto & 24 & 46,2 \\
\hline & Superior completo & 10 & 19,2 \\
\hline & Pós-graduação & 5 & 9,6 \\
\hline \multirow[t]{4}{*}{ Renda familiar } & Nenhuma renda & 1 & 1,9 \\
\hline & Até 1 salário mínimo & 25 & 48,1 \\
\hline & De 1 a 3 salários mínimos & 17 & 32,7 \\
\hline & De 3 a 6 salários mínimos & 9 & 17,3 \\
\hline
\end{tabular}

Fonte: Autores (2019).

Relacionado à quantidade de animais que cada entrevistado possuía, a maioria, $38(73,1 \%)$ possuíam de 1 a 3 animais e $14(26,9 \%)$ possuíam 4 animais ou mais (Figura 1). Dentre esses animais, 38 (73,1\%) dos entrevistados possuíam cão e gato e 14 (26,9\%) possuíam também outra espécie animal (Figura 2). Resultados semelhantes foram observados na mesma pesquisa realizada pelo SPC Brasil em 2017, onde 76\% dos entrevistados são donos de algum animal de estimação (SPC Brasil, 2017). De acordo com Gomes (2015), a maioria das pessoas tem preferência por criar unicamente cães, no entanto uma parte significativa dos tutores $(29,3 \%)$ possuíam mais de uma espécie animal. 
Figura 1. Quantidade de animais que os entrevistados possuíam.

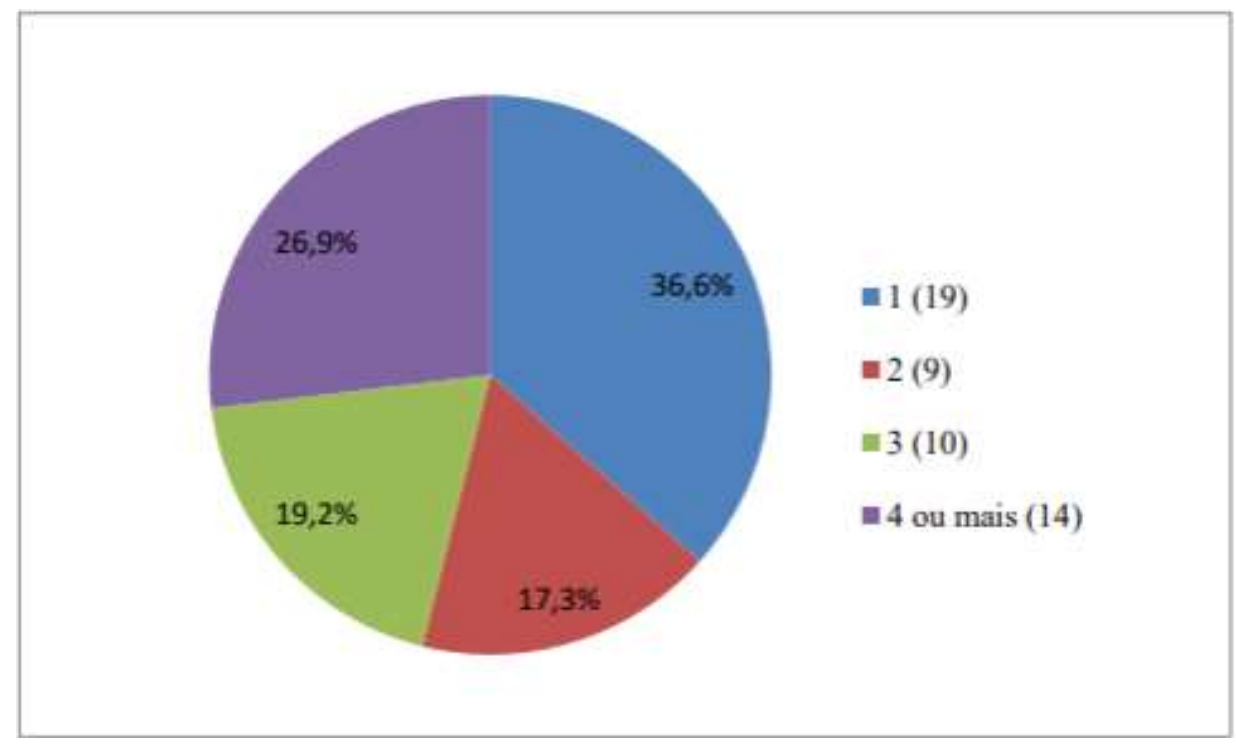

Fonte: Autores (2019).

Figura 2. Tipos de animais de estimação dos indivíduos entrevistados.

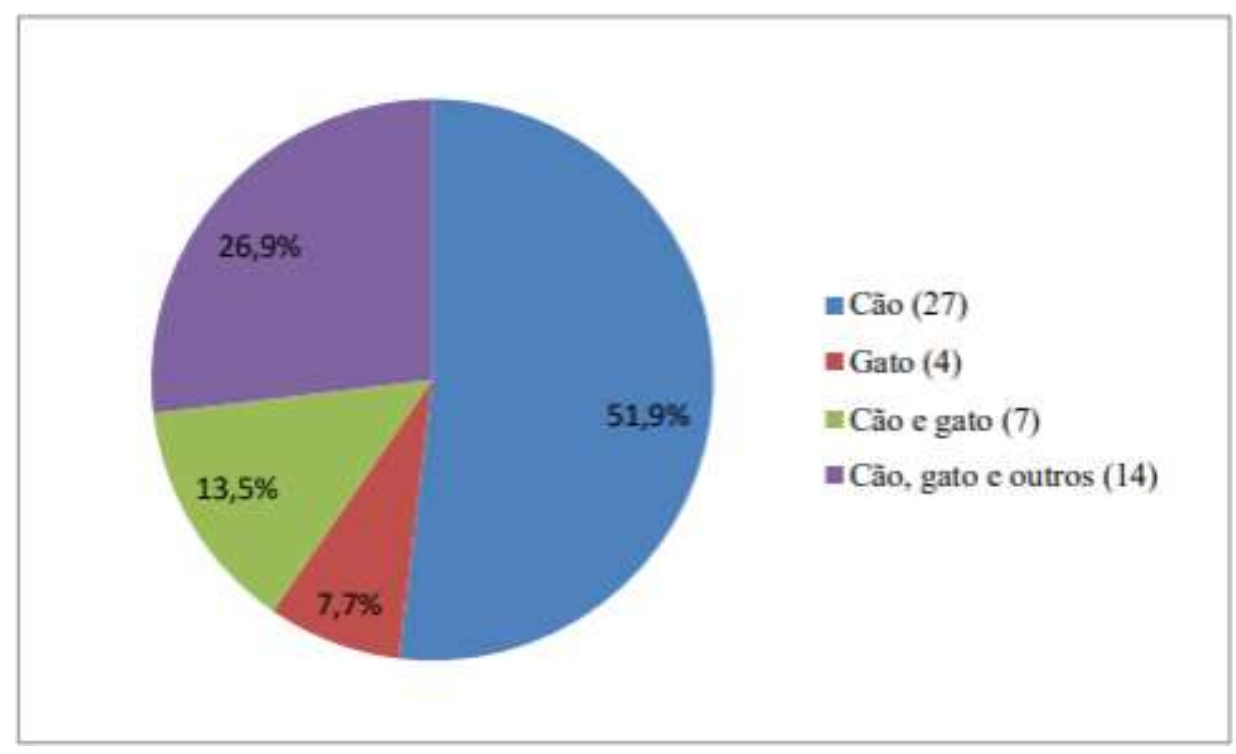

Fonte: Autores (2019).

Ainda na pesquisa realizada pelo SPC Brasil foi sugerido que as pessoas que convivem com algum animal de estimação apresentam uma relação extremamente afetiva com eles, assim tendo seus animais como amigos ou membros da família, capazes de corresponder todo o cuidado e atenção que recebem (SPC Brasil, 2017). Essa relação de membro da família sugere que esses animais podem ser submetidos a automedicação, prática bastante realizada por seres humanos, Zielke et al. (2018).

Quando questionados se já haviam medicado seus animais sem antes ir a um médico veterinário, 31 (59,6\%) responderam que sim e 21 (40,4\%) responderam que não (Figura 3). Segundo Benedito, Albuquerque, Taffarel e Bastos-Pereira (2017b), em uma pesquisa realizada na Universidade Estadual de Maringá, na clínica de pequenos animais, foi comprovado que 59\% dos animais já haviam sido medicados previamente à consulta. Outro estudo, realizado em Teresina - PI, confirma que $62,76 \%$ dos animais da região já haviam sido medicados sem orientação do profissional médico veterinário, seja por medicação laboratorial ou caseira, Quessada et al. (2010). 
Figura 3. Casos de automedicação em animais na cidade de Areia - PB.

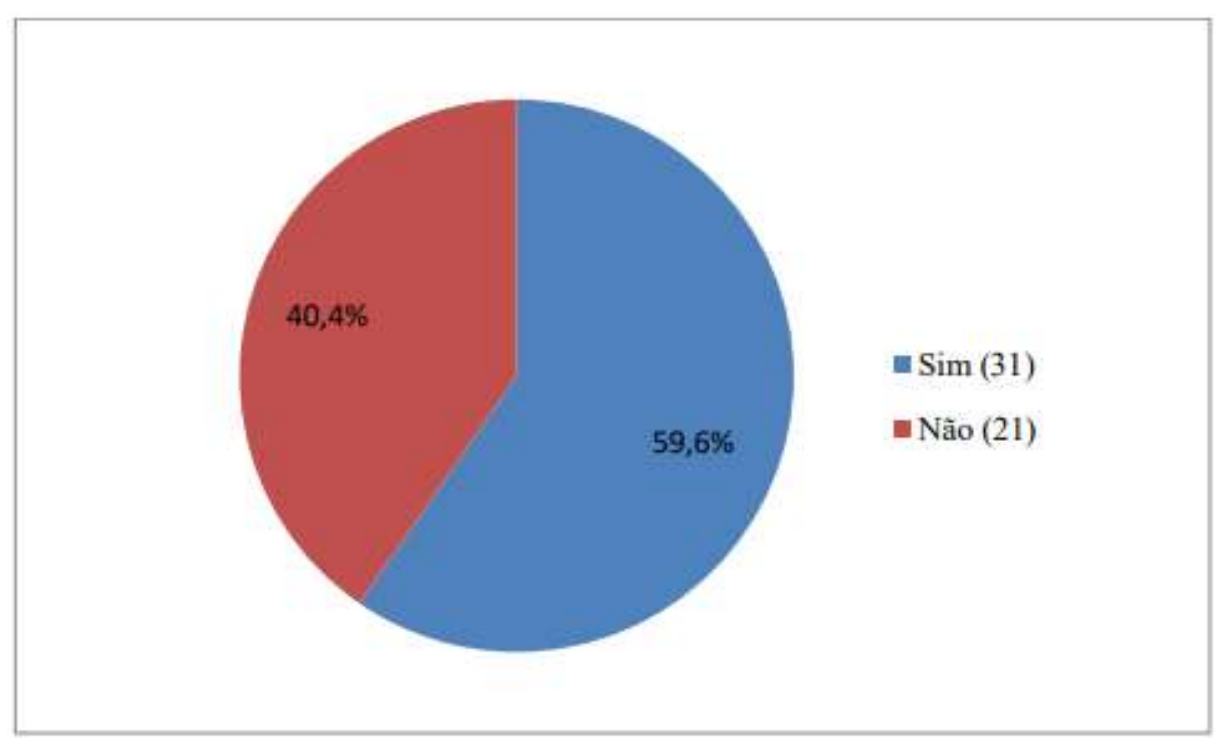

Fonte: Autores (2019).

Ao analisar os medicamentos mais utilizados pelos participantes que responderam positivamente o questionamento a respeito da medicação sem antes ir a um médico veterinário, a classe medicamentosa mais citada foi a dos AINES (dipirona, paracetamol, diclofenaco, meloxicam), correspondendo a 41,6\% (Figura 4). Dado semelhante ao encontrado por Mello, Gaira, Klein, Dalmolin e Pinto (2008), onde a classe dos anti-inflamatórios foi a mais utilizada, mas diferente dos achados de Carvalho, Araújo, Bonfim, Vieira e Azevedo (2012), em que os medicamentos mais utilizados foram os antibióticos. Segundo Riboldi, Lima e Dallegrave (2012), os animais são mais sensíveis aos AINES do que os humanos, grande parte das intoxicações em pequenos animais ocorre pela utilização dessa classe medicamentosa, devido à preocupação dos tutores em aliviarem os sinais clínicos do seu animal de estimação.

Figura 4. Classes medicamentosas utilizadas na automedicação em animais na cidade de Areia - PB.

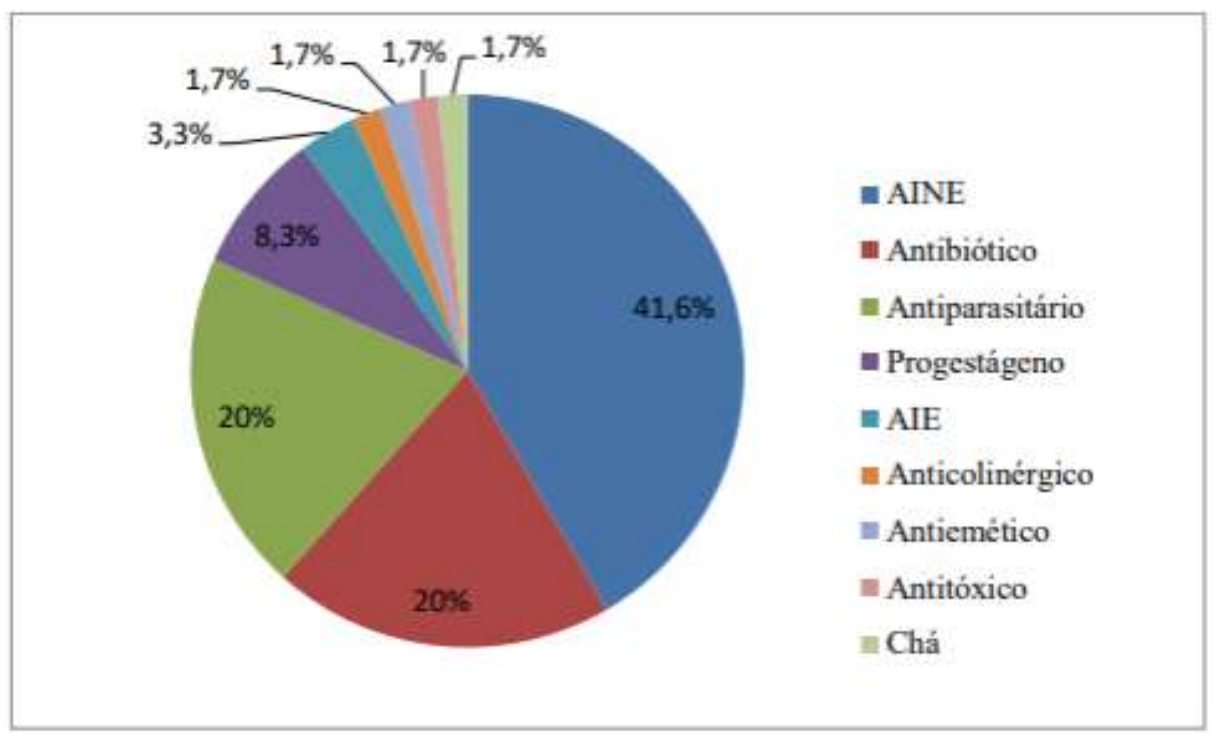

Fonte: Autores (2019). 
O uso dos antibióticos (amoxicilina, cefalexina, enrofloxacina, doxiciclina, sulfa+trimetoprim) teve prevalência de $20 \%$. Carvalho et al. (2012), aponta que pela facilidade de compra desses medicamentos, seja em farmácia humana ou veterinária, o hábito da automedicação com esses tipos de fármacos é frequente. No entanto, esse feito tem sido reduzido pela restrição da compra de antibióticos sem a apresentação de receituário médico ou médico veterinário, através da resolução nº20/2011 lançada pela ANVISA, Agência Nacional de Vigilância Sanitária [ANVISA] (2011).

A prevalência do uso dos antiparasitários (ivermectina) foi de $20 \%$. Um estudo realizado por Zielke et al. (2018), mostra dado semelhante, em que 19\% de caninos atendidos no Hospital de Clínicas Veterinárias da Universidade Federal de Pelotas foram tratados com antiparasitários. Frente ao uso indiscriminado de antiparasitários, essa também é uma causa comum de intoxicação medicamentosa em pequenos animais (Caloni, Cortinovis, Rivolta \& Davanzo, 2012). E em relação a ivermectina, é importante ressaltar que seu uso é restrito em cães das raças Collie, Shetland Sheepdog, Old English Sheepdog e Australian Sheepdog e em gatos, pois podem apresentar sinais de intoxicação (Almeida \& Ayres, 2011).

As outras classes medicamentosas citadas na pesquisa em questão foram os progestágenos, anti-inflamatórios esteroidais, anticolinérgicos, antieméticos, antitóxicos e chás, totalizando 18,4\%, conforme figuras 3 e 4 .

Foi também perguntado se os participantes conheciam os riscos da medicação sem a prescrição de um médico veterinário, a maioria (86,5\%) respondeu que sim (Figura 5). Dentre os riscos conhecidos pelos participantes, os mais citados foram vômito (26,5\%) e resistência bacteriana $(19,7 \%)$. Outros riscos citados pelos entrevistados foram diarreia sanguinolenta, convulsão, hemorragia, insuficiência renal, intoxicação e morte. O uso inadequado de algum medicamento pode fazer com que ocorram efeitos colaterais e/ ou efeitos tóxicos, que podem levar à morte. Salivação excessiva, diarreia, vômitos, sono profundo e exagerado, andar cambaleante, tremores e crises convulsivas são os primeiros sintomas diante de uma intoxicação medicamentosa, além disso, o uso indiscriminado de antibióticos é o causador de resistência bacteriana (Feldkircher, 2014; Dolce, 2014).

Figura 5. Conhecimento dos participantes a respeito dos riscos que a medicação sem a prescrição de um médico veterinário pode causar.

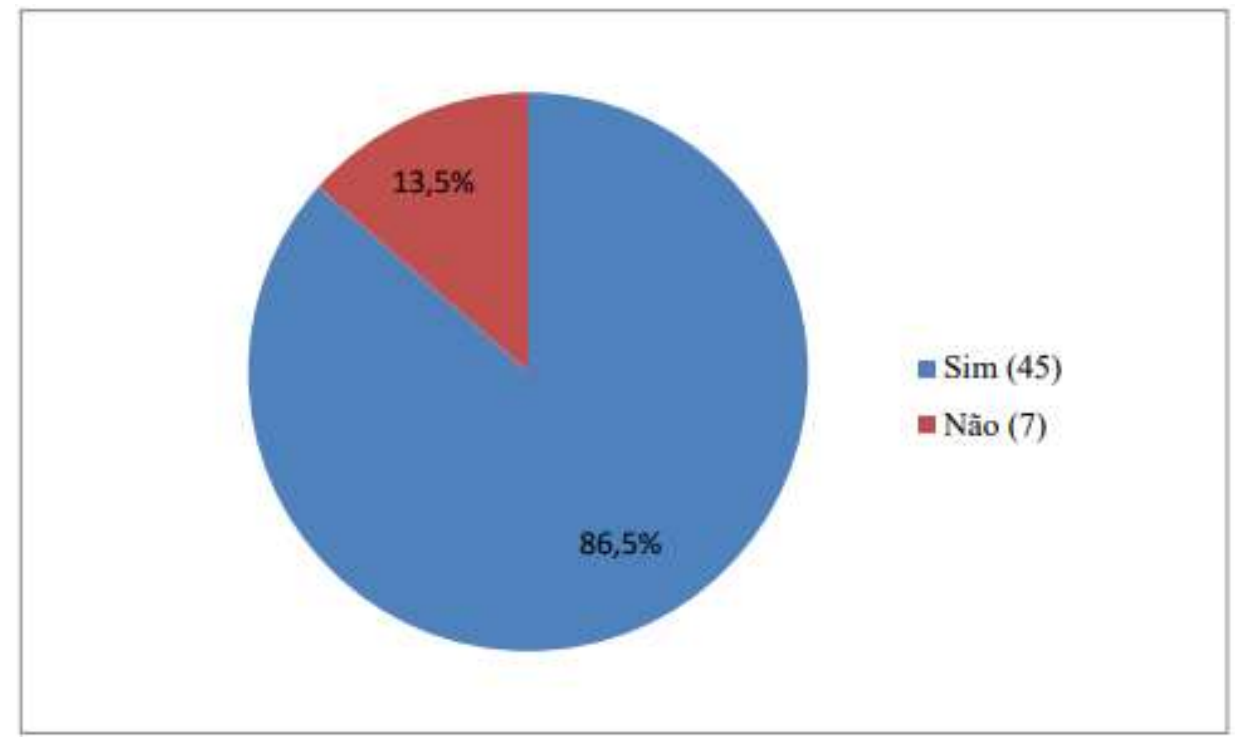

Fonte: Autores (2019).

Os participantes também foram questionados quanto ao conhecimento do potencial tóxico que alguns fármacos podem apresentar $34(65,4 \%)$ afirmaram conhecer (Figura 6). 
Figura 6. Conhecimento dos participantes a respeito do potencial tóxico que alguns medicamentos apresentam.

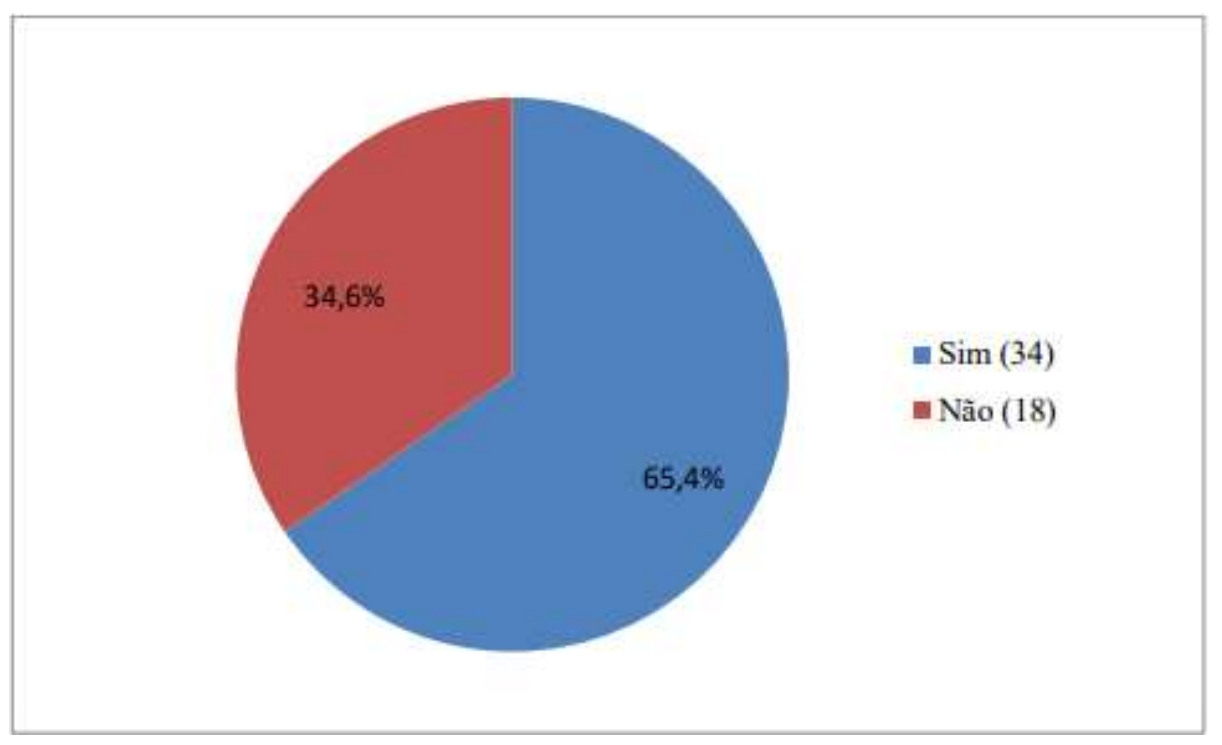

Fonte: Autores (2019).

Por fim, foi questionado se os entrevistados achavam correto medicar seus animais sem a orientação de um médico veterinário e $100 \%$ dos participantes, não acharam correto.

Apesar de grande parte dos entrevistados conhecerem os riscos da automedicação, relatarem de forma precisa as principais consequências do uso inadequado dos medicamentos e afirmarem conhecer o potencial tóxico que alguns fármacos apresentam, uma alta parcela dessas pessoas ainda realiza essa prática. E mesmo assim, também afirmam ser incorreto medicar animais sem a orientação de um médico veterinário. Diferente do resultado apresentado por Zielke et al. (2018) onde 22\% dos participantes da pesquisa acreditavam que alguns medicamentos podem ser utilizados por conta própria e que não fazem nenhum mal.

A facilidade em adquirir medicamentos sem a orientação de um profissional e a ansiedade do tutor em resolver o problema que o animal apresenta e aliviar os sinais clínicos, pode explicar o motivo dessa prática ainda ser realizada com frequência, Carvalho et al. (2012); Zielke et al. (2018). Outro motivo para que a automedicação ocorra é que muitas vezes o tutor não deseja desembolsar recursos com consultas ou exames, que são os meios indicados para resolver o problema que o animal apresenta.

\section{Conclusão}

A realização dessa pesquisa propiciou a obtenção de mais conhecimento sobre o tema estudado, visto que os dados referentes à prática da medicação em animais de companhia sem a orientação do médico veterinário, ainda são escassos. Também foi possível identificar os fármacos comumente utilizados, o nível de conhecimento da população a respeito dos riscos da automedicação e o potencial tóxico que alguns fármacos apresentam. Grande parte dos participantes afirmaram utilizar medicamentos que os mesmos utilizariam para si, e também relataram conhecer os riscos e consequências da automedicação, no entanto, uma parcela considerável ainda realiza a prática. O que confirma a necessidade de mais esclarecimento à população $\mathrm{e}$ leis em defesa da saúde animal. A prática da medicação sem a prescrição do médico veterinário pode trazer consequências graves para o animal, sendo assim de grande importância a realização de pesquisas como esta. Consequentemente, esse estudo contribuirá para que novos trabalhos na área sejam realizados e para promoção de ações em favor da saúde animal. 


\section{Referências}

Aaker, D., Kumar, V. \& Day, G. (1995). Marketing research. John Wiley \& Sons, Inc.

Almeida, M. A. O., Ayres, M. C. C. (2011). Agentes Antinematódeos. In Spinosa, H. S., Górniak, S. L., Bernardi, M. M. Farmacologia Aplicada à Medicina Veterinária. (517-530). Guanabara Koogan.

Anjos, T. M. \& Brito, H. F. V. (2009). Terapêutica felina: diferenças farmacológicas e fisiológicas. Medvep - Revista Científica de Medicina Veterinária Pequenos Animais e Animais de Estimação. 7(23), 554-567.

Agência Nacional de Vigilância Sanitária. (2007). Projeto Educação e Promoção da Saúde no Contexto Escolar: o contributo da Agência Nacional de Vigilância Sanitária para o uso racional de medicamentos. https://www.gov.br/anvisa/pt-br/centraisdeconteudo/publicacoes/educacao-e-pesquisa/publicacoes-sobreeducacao-e-pesquisa/caderno-do-professor-promocao-da-saude-no-contexto-escolar.pdf/@@downl oad/file/Caderno\%20 do\%2 0pr ofessor\%20_\%20Pr omo $\%$ C3\% A3o\%20da $\% 20 \mathrm{Sa} \% \mathrm{C} 3 \%$ BAde $\% 20$ no $\% 20$ Contexto $\% 20$ Escolar.pdf

Agência Nacional de Vigilância Sanitária. (2011). Informe Técnico Sobre a RDC N²0/2011. https://www.anvisa.gov.br/SN GPC/Informe_Tecnico_Procedimentos_RDC_n_20.pdf?WCM_PORTLET=PC_7_CGAH47L00G1870I8G5FBUC30V1_WCM\&WCM_GLOBAL_CONTE $\mathrm{XT}=/ \mathrm{wps} / \mathrm{wcm} /$ connect/anvisa/anvisa/informes\%2Btecnicos/publicacao\%2Binformes $\% 2 \mathrm{Btecnicos} / \mathrm{sngpc} \% 2 \mathrm{Binforme} \% 2 \mathrm{Btecnico} \% 2 \mathrm{Bpara} \% 2 \mathrm{Bharmonizacao}$ $\% 2 \mathrm{Bdos} \% 2 \mathrm{~B}$ procedimentos $\% 2 \mathrm{Bda} \% 2 \mathrm{Brdc} \% 2 \mathrm{Bn} \% 2 \mathrm{~B} 20 \% 2 \mathrm{~B} 2011$

Benedito, G. S., Albuquerque, A. P. L., Taffarel, M. O. \& Bastos-Pereira, A. L. (2017a). Incidência de medicação sem prescrição em um hospital veterinário na cidade de Umuarama, Paraná, no período entre 2011 e 2015. Revista de Ciência Veterinária e Saúde Pública. 5(2), $140-157$.

Benedito, G. S., Albuquerque, A. P. L., Taffarel, M. O. \& Bastos-Pereira, A. L. (2017b, maio). Medicação sem prescrição em animais de companhia: como prevenir? Anais do II Simpósio em Produção Sustentável e Saúde Animal, Umuarama, PR, Brasil.

Caloni, F., Cortinovis, C., Rivolta, M. \& Davanzo, F. (2012). Animal poisoning in Italy: 10 years of epidemiological data from the poison Control Centre of Milan. Veterinary Record. 170(16), 415.

Carvalho, C. F., Araújo, D. P., Bonfim, J. C., Vieira, D. F. \& Azevedo, J. C. (2012). Incidência de medicação em cães e gatos por seus responsáveis sem orientação médico-veterinária: levantamento em um hospital veterinário universitário. Enciclopédia Biosfera - Centro Científico Conhecer. 8(15), 1035 - 1042.

Dolce, V. B. H. (2014). Prevalência de “automedicação" descrita na anamnese em cães e gatos atendidos no Hospital Veterinário da Universidade de Cuiabá/MT. Dissertação, Universidade de Cuiabá, Cuiabá, MT, Brasil.

Feldkircher, K. C. G. (2014). Intoxicação medicamentosa em animais domésticos. REVET - Revista Científica de Medicina Veterinária - FACIPLAC. 1(1), 1418 .

Fundação Getúlio Vargas Social. (2014). Qual a faixa de renda familiar das classes? https://cps.fgv.br/qual-faixa-de-renda-familiar-das-classes

Gomes, V. C. P. S. (2015). Relação entre padrão socioeconômico e variáveis ligadas ao bem estar e guarda responsável de cães e gatos em Areia - PB. Trabalho de Conclusão de Curso, Universidade Federal da Paraíba, Areia, PB, Brasil.

Instituto Brasileiro de Geografia e Estatística. (2020). Pesquisa Nacional de Saúde 2019. https://biblioteca.ibge.gov.br/visualizacao/livros/liv101748.pdf

Mello, F. P. S., Gaira, M. S., Klein, N., Dalmolin. F. \& Pinto,S. T. L., Fi. (2008, outubro). Incidência de Automedicação em Cães e Gatos atendidos no Hospital Veterinário da Puc-RS de Julho de 2007 a Junho de 2008. Anais do XXVIII Congresso Brasileiro de Medicina Veterinária, Gramado, RS, Brasil.

Mota, J. S. (2019). Utilização do Google Forms na pesquisa acadêmica. Revista Humanidades e Inovação. 6(12), 371-380.

Quessada, A. M., Carvalho, R. L., Klein, R. P., Silva, F. A. N., Fonseca, L. S., Miranda, D. F. H. \& Souza Júnior, S. C. (2010). Uso de medicamentos sem prescrição médico-veterinária-comunicação. Vet. Not. 16(1), 69-71.

Riboldi, E. O. (2010). Intoxicações em pequenos animais: uma revisão. Trabalho de Conclusão de Curso, Universidade Federal do Rio Grande do Sul, Porto Alegre, RS, Brasil.

Riboldi, E., Lima, D.A. \& Dallegrave, E. (2012). Sensibilidade espécie-específica aos anti-inflamatórios não esteroidais: humanos X animais de companhia. Arq. Bras. Med. Vet. Zootec. 64(1), 39-44.

Santos, D. R. (2014). Perfil da automedicação no Brasil. Trabalho de Conclusão de Curso, Faculdade Maria Milza, Governador Mangabeira, BA, Brasil.

Tasaka, A. C. (2011). Anti-inflamatórios não esteroidais. In Spinosa, H. S., Górniak, S. L., Bernardi, M. M. (Ed.). Farmacologia Aplicada à Medicina Veterinária. (5, 245-259). Guanabara Koogan.

Xavier, F. G., Maruo, V. M., Spinosa, H. S. (2008). Toxicologia dos medicamentos. In Spinosa, H. S., Górniak, S. L., Palermo-Neto, J. (Ed.). Toxicologia Aplicada à Medicina Veterinária. (1, 117-190). São Paulo: Manole.

Zielke, M., Carvalho, L. F., Salame, J. P., Barboza, D. V., Gaspar, L. F. J. \& Sampaio, L. C. L (2018). Avaliação do uso de fármacos em animais de companhia sem orientação profissional. Science And Animal Health - Faculdade de Veterinária e Programa de Pós Graduação em Veterinária da Universidade Federal de Pelotas. 6(1), 29-46.

Serviço de Proteção ao Crédito Brasil. (2017). Mercado de consumo pet. https://www.spcbrasil.org.br/wpimprensa/wp-content/uploads/2017/09/Ap resentacao_Mercado_Pet_Setembro_2017.pdf 